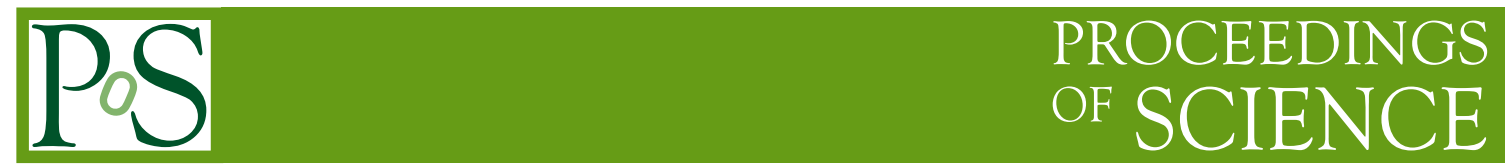

\title{
Recent Progress in Lattice QCD Thermodynamics
}

\author{
Carleton DeTar* \\ Physics Department, University of Utah, Salt Lake City, UT 84112, USA \\ E-mail: detar@physics.utah.edu
}

\begin{abstract}
This review gives a critical assessment of the current state of lattice simulations of QCD thermodynamics and what it teaches us about hot hadronic matter. It outlines briefly lattice methods for studying QCD at nonzero temperature and zero baryon number density with particular emphasis on assessing and reducing cutoff effects. It discusses a variety of difficulties with methods for determining the transition temperature. It uses results reported recently in the literature and at this conference for illustration, especially those from a major study carried out by the HotQCD collaboration.
\end{abstract}

The XXVI International Symposium on Lattice Field Theory

July 14 - 19, 2008

Williamsburg, Virginia, USA

\footnotetext{
* Speaker.
} 


\section{Introduction}

At an early stage the universe was very likely a quark-gluon plasma. In heavy ion colliders we seek to recreate this state of matter and study its properties. Lattice gauge theory is ideally suited for the fully nonperturbative study of quantum chromodynamics under conditions close to thermal equilibrium. The insights gained from lattice simulations can be extrapolated through hydrodynamic modeling to the quasi-equilibrium expansion of the plasma. In this way lattice calculations provide crucial assistance in the interpretation of experimental results [1].

The baryon density was essentially zero in the early universe. At high densities, even at low temperature, popular tradition predicts that hadronic matter is also in a deconfined plasma state. At still higher densities even more unusual phases, such as a color-flavor locked phase have been proposed [2]. For technical reasons, such high densities are beyond the reach of lattice simulations using standard methods. At least we can hope to simulate matter at the low densities found in heavy ion collisions.

In this talk I consider only progress in calculations at zero baryon number density. In the companion talk Shinji Ejiri describes developments in nonzero density calculations [3].

Here are highlights of recent advances at zero baryon density, which I will cover in this talk:

- HotQCD study. A high statistics study is being carried out on the IBM BlueGene at the Lawrence Livermore National Laboratory. This study compares results in closely matched simulations from two staggered fermion actions, namely asqtad and $\mathrm{p} 4 \mathrm{fat} 3$, and it is providing the first large-scale simulation at $N_{\tau}=8$ with domain wall fermions. (See talks by M. Cheng [4], R. Gupta [5], and W. Soeldner [6].)

- Chiral susceptibility. New insights into the behavior of the chiral susceptibility will change the determination of $T_{c}$ using this quantity [7].

- Equation of state. A new method has been proposed [8].

- Transport coefficients. There are new ideas and methods for computing them [9].

- Spatial string tension. A new result agrees surprisingly well with 3D perturbation theory [10].

I will not have time to cover interesting studies of QCD-like theories with a large number of flavors [11]. And I regret that time and space did not permit covering all recent work in this field.

I will try to give a general and fairly critical overview, using selected results from the parallel sessions as illustrations and leaving the details to the parallel sessions. After a brief review of lattice methodology I discuss potential cutoff problems with various actions and focus on issues and confusion in determining $T_{c}$. Turning to results, I highlight some new methods and results for the equation of state, allude to recent progress in determining transport coefficients, and end with mention of a little surprise concerning predictions of dimensional reduction for the spatial string tension. 


\section{Lattice Methodology}

Lattice methods are especially well suited for simulating a quantum statistical ensemble in thermal equilibrium at fixed temperature $T$. We set a finite imaginary time interval

$$
a N_{\tau}=1 / T
$$

for lattice spacing $a$ and $N_{\tau}$ sites in imaginary time, and we impose periodic (antiperiodic) boundary conditions on the bosonic (fermionic) fields. Under these conditions the lattice Feynman path integral generates the quantum partition function for the underlying hamiltonian $H$,

$$
Z=\operatorname{Tr} \exp (-H / T)
$$

in the continuum limit. Operator expectation values are thermal expectation values in this ensemble. Since simulations with standard methods are limited to equilibrium and near-equilibrium processes, to apply lattice results to the nonequilibrium conditions of heavy ion collisions requires phenomenological modeling.

The temperature is varied by changing either $N_{\tau}$ or $a$. The latter strategy is more common. At fixed $N_{\tau}$, decreasing the gauge coupling $g^{2}$ decreases $a$, so $T$ grows. It is common now to adjust the bare lattice quark masses together with the lattice spacing so that zero temperature meson masses remain fixed. In this way variations in observables can be attributed to changes in temperature and not also to changes in the Hamiltonian. Such trajectories through parameter space are called "lines of constant physics."

Of course, to connect with reality we need also to take the continuum limit. With the fixed $N_{\tau}$ strategy, the lattice is coarser at low temperatures and finer at high temperatures. For a given temperature, obviously, we approach the continuum by repeating the calculation at smaller $a$ and larger $N_{\tau}$. Contemporary lattice simulations have $N_{\tau}$ as large as 12 for some quantities [12], but 6 and 8 are typical for expensive quantities such as the equation of state. Table 1 shows the relationship between lattice spacing and $N_{\tau}$ at $T=180 \mathrm{MeV}$, near the crossover temperature for QCD. We see that by standards of contemporary zero temperature simulations, thermodynamic simulations at $N_{\tau}=6$ and 8 are rather coarse at this temperature, and $N_{\tau}=4$ is extremely coarse. Thus we must be alert to the possibility of distortions due to cutoff effects.

\begin{tabular}{|l|l|l|l|l|l|}
\hline$N_{\tau}$ & 4 & 6 & 8 & 10 & 12 \\
\hline$a(\mathrm{fm})$ & 0.27 & 0.18 & 0.14 & 0.11 & 0.09 \\
\hline
\end{tabular}

Table 1: Lattice spacing $v s . N_{\tau}$ at $T=180 \mathrm{MeV}$.

The continuum limit can be expensive. For the equation of state the computational cost grows with decreasing lattice spacing as $a^{-11}$. This places a high premium on reducing undesirable cutoff effects at a coarse lattice spacing. The degree of "improvement" of the lattice action is a significant factor.

The most extensive recent simulations use staggered fermions with varying degrees of improvement. The asqtad staggered formalism is designed to eliminate cutoff effects at $\mathscr{O}\left(a^{2}\right)$, leaving errors at $\mathscr{O}\left(\alpha_{s} a^{2}\right)$ (see references in [13]). The p4fat3 staggered action is also improved, but 


\begin{tabular}{|c|c|c|c|}
\hline action & $A_{2} / A_{0}$ & $A_{4} / A_{0}$ & $A_{6} / A_{0}$ \\
\hline \hline standard staggered & $248 / 147$ & $635 / 147$ & $3796 / 189$ \\
\hline Naik & 0 & $-1143 / 980$ & $-365 / 77$ \\
\hline $\mathrm{p} 4$ & 0 & $-1143 / 980$ & $73 / 2079$ \\
\hline \hline standard Wilson & $248 / 147$ & $635 / 147$ & $13351 / 8316$ \\
\hline hypercube & -0.242381 & 0.114366 & -0.0436614 \\
\hline \hline $\begin{array}{c}\text { overlap/ } \\
\text { domain wall }\end{array}$ & $248 / 147$ & $635 / 147$ & $3796 / 189$ \\
\hline
\end{tabular}

Table 2: Continuum limit scaling behavior of free massless quarks in various lattice formulations, based on an expansion (2.3) of the pressure in powers of $1 / N_{\tau}^{2}$ from [16]. Shown are ratios of the expansion coefficients to the ideal, leading Stefan-Boltzmann coefficient. A small ratio indicates good scaling.

it does not eliminate all such effects [14]. Both actions also improve the free-quark dispersion relation, which is desirable in a high temperature deconfined environment. The Budapest-Wuppertal action with stout gauge links and unimproved staggered fermions does not improve the dispersion relation [15], but it does reduce taste-splitting effects.

Improvement is good, but one may carry it too far. Improvement tends to fatten action operators, in which case localization could become an issue. It is plausible that the extent to which a lattice simulation approximates the quantum partition function (2.2) depends on the locality of the lattice transfer matrix. Ideally the localization length of the action $\ell$ should be much less than $1 / T$ or in lattice units, much less than $N_{\tau}$.

For free fermions cutoff effects for various lattice formulations can be studied analytically. Recently Hegde et al. [16] looked at deviations from the expected free-fermion Stefan-Boltzmann relation for the pressure $p$ as a function of $1 / N_{\tau}^{2}$ (equivalently $a^{2}$ ) and chemical potential $\mu / T$ :

$$
\frac{p}{T^{4}}=\sum_{k=0}^{\infty} A_{2 k} P_{2 k}(\mu / \pi T)\left(\frac{\pi}{N_{\tau}}\right)^{2 k}
$$

The leading term $A_{0}$ is the Stefan-Boltzmann term. The ratios of higher coefficients $A_{2 k} / A_{0}$ measure the strength of the cutoff effects. These terms measure the ability of the action to approximate the continuum free fermion dispersion relation. Table 2 reproduces their results for a variety of actions. We see that the hypercube action [17] has pleasingly small coefficients. The Naik (asqtad) and $\mathrm{p} 4$ (p4fat3) actions remove the second order term as designed, but the $\mathrm{p} 4$ action is better at sixth order. The standard (unimproved) staggered action (regardless of gauge-link smearing) does as poorly as the standard (and clover-improved) Wilson actions. The overlap and domain wall actions constructed from the standard Wilson kernel inherit its poor behavior.

In selecting a fermion action for thermodynamics, there should be no excuse for deliberately building in poor continuum scaling.

Another recent study confirms pronounced cutoff problems with free chiral fermion actions based on the standard Wilson kernel. Gavai and Sharma calculated the ratio of the lattice energy density to the expected Stefan-Boltzmann value for overlap and domain wall fermions [18]. Their 

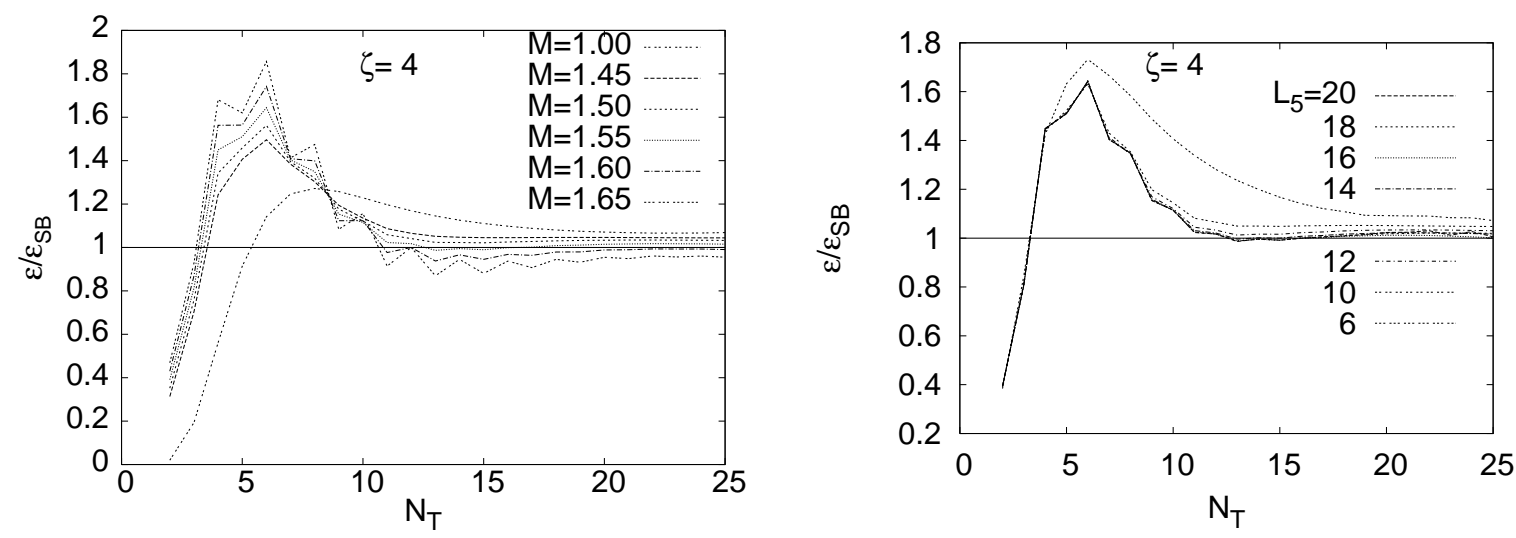

Figure 1: Deviation of the lattice free quark energy density from the Stefan-Boltzman continuum energy density as a function of $N_{\tau}$ from [18]. ( $\left.\zeta=N_{s} / N_{\tau}=4\right)$. Left panel: overlap for various mass shifts $M$. Right panel: domain wall fermions for various $L_{S}$ at $M=1.55$.

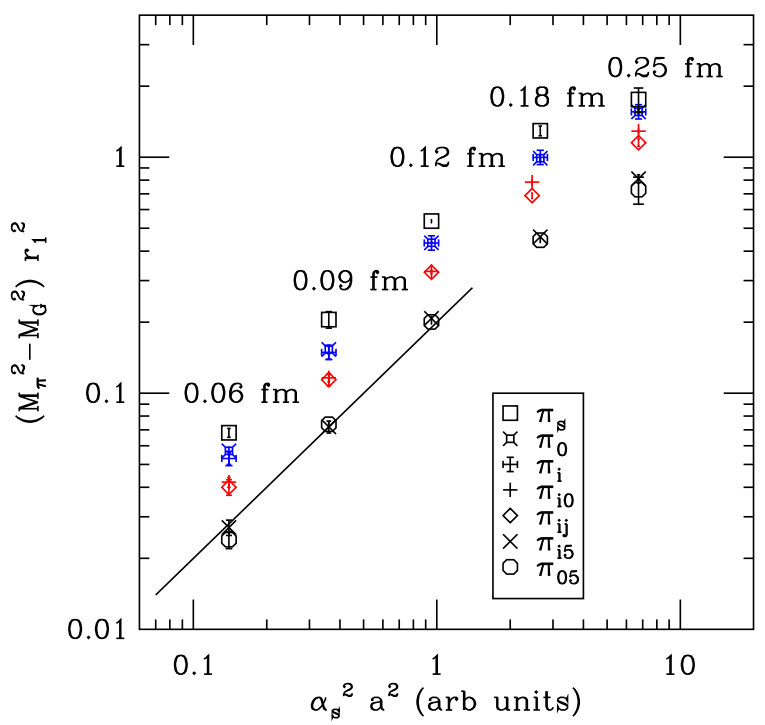

Figure 2: Splitting of the pion taste multiplet $v s . \alpha_{s}^{2} a^{2}$ for a wide range of lattice spacings. Splitting is measured as the difference of the squared masses of the member and the Goldstone member. The plot symbols distinguish the members of the multiplet. The line is drawn with unit log-log slope to test proportionality to $\alpha_{s}^{2} a^{2}$.

results, reproduced in Fig. 1, show slow continuum scaling and an oscillation related to negative eigenvalues of the transfer matrix.

Staggered fermions have the awkward problem of extra "taste" degrees of freedom. The standard "fourth root" trick gives an approximately correct counting of species, but hadrons in the statistical ensemble still come in multiplets with a range of masses. This is especially visible in the pion spectrum. The splitting of taste multiplets is predicted to decrease as $\mathscr{O}\left(a^{2} \alpha_{s}^{2}\right)$ in terms of lattice spacing and color fine structure constant $\alpha_{s}$. Figure 2 confirms this trend for the pion taste multiplet as a function of lattice spacing. 
In judging how closely a simulation comes to the physical point, devotees of staggered fermions may be tempted to focus on the lowest member of the pion multiplet, i.e., the Goldstone boson pion. While this practice is correct when the Goldstone boson can be an isolated external state, as in a zero temperature Green's function, in a thermal ensemble, all members of the multiplet participate. Thus it is more appropriate in thermodynamics simulations to compare an average multiplet mass, e.g., the rms pion mass with the physical pion mass. The physical point is reached only by reducing the lattice spacing together with the light quark mass. It is incorrect to claim a thermodynamics calculation is done at a physical pion mass when the rms mass is still much higher.

Is taste-symmetry breaking really a problem for thermodynamics? It is believed to be most dramatic for the pion and less noticeable for more massive states [19]. One could argue that close to the crossover temperature away from the critical point, so many excited states participate, as in the resonating hadron gas model, that pions do not matter much. But if approach the critical point at fixed lattice spacing, taste splitting is likely to have a strong effect on the critical behavior: we may even get a chiral symmetry restoring transition in the wrong universality class. And certainly at quite low temperatures where pions dominate the statistical ensemble, taste splitting makes a difference.

\section{Phase Diagram and Determination of $T_{c}$}

\subsection{Current consensus}

Is there a genuine phase transition separating a low temperature confined phase with spontaneously broken chiral symmetry from a high temperature deconfined phase with restored chiral symmetry? The answer depends on the number of quark flavors and their masses. Figure 3 sketches the current qualitative theoretical consensus for the case of $2+1$ flavors of quarks with masses $m_{u}=m_{d}$ and $m_{s}$ [20]. In the upper right corner the quark masses are so large they play no role in the statistical ensemble, and we enter the well-studied regime of a first order confining/deconfining transition in pure Yang-Mills theory. At low temperature chiral models predict a first order transition for degenerate masses, shown in the lower left cormer, and a second order transition at large $m_{s}$ when $m_{u}=m_{d}=0$ (provided the chiral anomaly does not vanish at the transition) [21]. ${ }^{1}$ The low-mass first order region is bounded by a critical line above which the transition is only a crossover.

Figure 3 is only qualitative. To say whether there is a phase transition at physical quark masses requires numerical simulation. The long-standing consensus has been that it is only a crossover. Aoki et al. have made a strong case for this conclusion [24]. Locating the actual critical line is challenging, since it occurs at small quark masses and is quite sensitive to cutoff effects [25, 26]. DeForcrand and Philipsen [27, 28] have done an impressively high statistics study that maps out the phase boundary, but only with unimproved staggered fermions at $N_{\tau}=4$. They have begun work at $N_{\tau}=6[28]$.

\footnotetext{
${ }^{1}$ In contradiction to this expectation, D'Elia, Di Giacomo, and Pica found indications of a first-order transition using an unimproved staggered fermion action and $N_{\tau}=4$ [22]. It is important to check this conclusion with a more refined action. In support of this expectation, Kogut and Sinclair have found a second order phase transition, but in the $\mathrm{O}(2)$ universality class, rather than $\mathrm{O}(4)$ [23].
} 


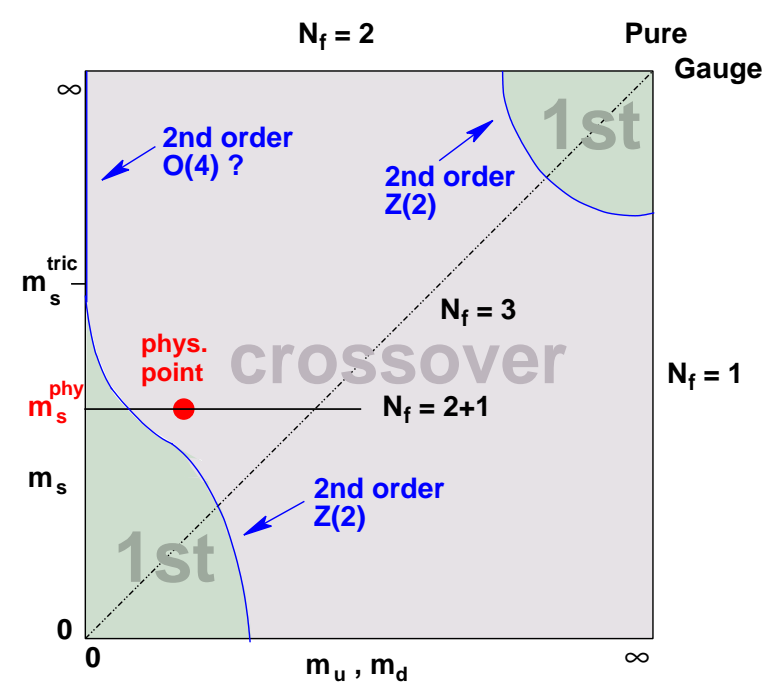

Figure 3: Sketch from [20] of the phase diagram for QCD at zero baryon density in $2+1$ flavor QCD as a function of the light quark masses showing regions where a high temperature phase transition or crossover is expected. For a second-order phase transition, the universality class is shown. Whether the expected tricritical strange quark mass $m_{s}^{\text {tric }}$ is higher or lower than the physical strange quark mass $m_{s}^{\text {phys }}$ is not yet firmly established.

\subsection{How precisely can we know $T_{c}$ ?}

So what is the crossover temperature $T_{c}$ ? One must ask, first, for what purpose do we need to know it? If there is only a crossover, the determination of $T_{c}$ is unquestionably imprecise, as the Budapest-Wuppertal group has emphasized [29]. For phenomenology it should be good enough to determine the temperature range over which an interesting quantity, such as the energy density changes rapidly. Each observable may give a somewhat different answer.

For (presumably) unphysical quark masses for which a genuine phase transition occurs, the transition temperature $T_{c}$ is unambiguous and precision is achievable. The observables used to locate it must, obviously, have a sensible continuum limit, and they should expose the critical behavior.

Finally, in determining the temperature there is a related question of setting the lattice scale. On coarse lattices, the result depends strongly on the physical quantity used to set the scale.

With these preliminaries in mind we examine a variety of observables that have been used to determine the crossover temperature. First we consider "deconfinement-type" observables.

\subsection{Deconfinement observables}

\subsubsection{Strange quark number susceptibility and equation of state}

The strange quark number susceptibility measures fluctuations in the strange quark number:

$$
\chi_{s}=\left\langle N_{s}^{2}\right\rangle /(V T)
$$

The energy density and pressure are also good indicators of the progress of deconfinement.

Recent results from the HotQCD collaboration are shown in Fig. 4 [5, 30]. 

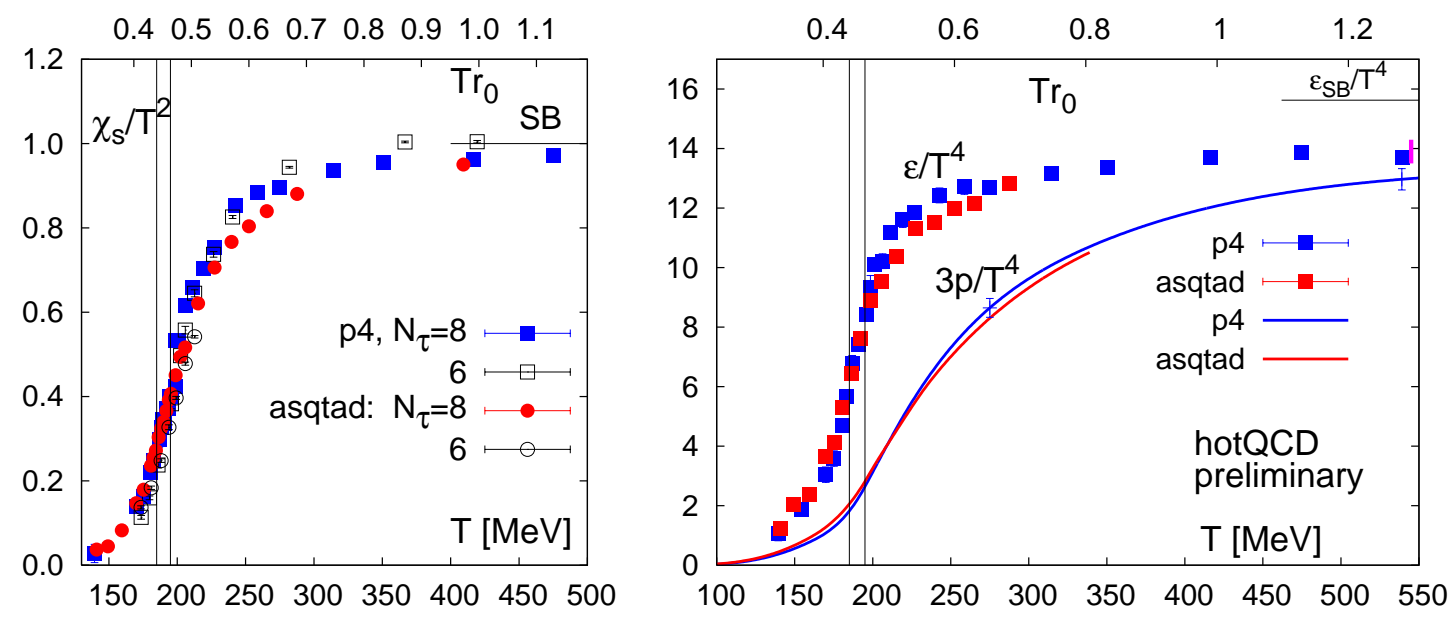

Figure 4: Left panel: Strange quark number susceptibility divided by the square of the temperature $v s$. temperature in $\mathrm{MeV}$ units (bottom scale) and $r_{0}$ units (top scale) for $N_{\tau}=6$ and 8. Right panel: equation of state showing energy density and three times the pressure, both divided by the fourth power of the temperature $v s$. temperature for $N_{\tau}=8$. Measurements are taken along a line of constant physics with $m_{u d}=0.1 m_{s}$. These preliminary results are from a HotQCD study comparing p4fat3 and asqtad staggered fermion formulations [5, 30]. The blue error bars on the pressure curve indicate the size of the error. The magenta bar shows a systematic error from setting the lower limit of the pressure integration. The vertical bands here and in HotQCD figures below indicate a temperature range 185 - $195 \mathrm{MeV}$ and serve to facilitate comparison.

We note that the asqtad and $\mathrm{p} 4 \mathrm{fat} 3$ results are in fair agreement. We see that there are dramatic changes in both observables over the temperature range $180-200 \mathrm{MeV}$. The scale has been set through the Sommer parameter $r_{0}$ (or $r_{1}$ ), which, in turn, is calibrated by the measured splitting in bottomonium [31]. The rough agreement between the $N_{\tau}=6$ and 8 gives some support for the utility of this scale determination.

\subsubsection{Universal critical behavior}

To determine the crossover temperature more precisely, one may look for the inflection point in the quark number susceptibility or a peak in specific heat. As we have remarked, the result is unambiguous only for a genuine phase transition. Both of these quantities are derivatives of the free energy $f=-T \log Z$, which leads to a unified treatment of their critical behavior [32]. At a critical point, the free energy can be decomposed into singular and analytic contributions. The singular part scales according to [32, 33]

$$
f_{s}\left(T, \mu_{q}\right)=b^{-1} f_{s}\left(t b^{1 /(2-\alpha)}\right) \sim t^{2-\alpha}
$$

where from charge symmetry and analyticity, the scaling variable depends on temperature and chemical potential through

$$
t=\left|\frac{T-T_{c}}{T_{c}}\right|+c\left(\frac{\mu_{q}}{T_{c}}\right)^{2} .
$$

For $O(4)$ the critical exponent is $\alpha \approx-0.25$. This relation can be used to predict singularities in quantities expressible as derivatives of the free energy. At $\mu_{q}=0$ the light quark number suscepti- 
bility is

$$
\chi_{\ell} / T^{2} \sim \frac{\partial^{2} f_{s}}{\partial \mu^{2}} \sim t^{1-\alpha},
$$

The singularity is weak and masked by analytic contributions. Its temperature derivative has a stronger singularity

$$
\frac{\partial\left(\chi_{\ell} / T^{2}\right)}{\partial T} \sim t^{-\alpha} .
$$

The same leading singularity is found in the specific heat

$$
C_{V} \sim \frac{\partial^{2} f_{s}}{\partial T^{2}} \sim t^{-\alpha}
$$

and in the quartic quark number susceptibility $c_{4}^{q}=\left(\left\langle N_{q}^{4}\right\rangle-3\left\langle N_{q}^{2}\right\rangle\right)$ :

$$
c_{4}^{q} \sim \frac{\partial^{4} f_{s}}{\partial \mu_{q}^{4}} \sim t^{-\alpha}
$$

\subsubsection{Screening free energy of a static quark}

The Polyakov loop measures the screening free energy of a static quark [34]. Thus it is a phenomenologically interesting deconfinement-type observable. It is not the derivative of the free energy, so it might not reveal critical behavior. It is customary to renormalize it by removing a temperature-independent self-energy [35], leaving

$$
F_{q}(T)=-T \log \left[P_{\text {renorm }}(T)\right]
$$

This quantity is shown in Fig. 5. The related susceptibility (essentially the variance in this quantity) is sometimes used to locate the transition temperature, but in numerical simulation the peak in this susceptibility weakens with increasing $N_{\tau}$.

\subsection{Chiral observables}

Next, we consider observables usually associated with a chiral phase transition.

\subsubsection{Chiral condensate}

The chiral condensate has an ultraviolet singularity for nonzero quark mass and (at least for $N_{f}=2$ ) an infrared chiral singularity at zero quark mass $m_{u d}$ :

$$
\langle\bar{\psi} \psi\rangle\left(a, m_{u d}, T\right) \sim \begin{cases}c_{1 / 2}(a, T) \sqrt{m_{u d}}+c_{1} m_{u d} / a^{2}+\text { analytic } & T<T_{c} \\ c_{1} m_{u d} / a^{2}+c_{\delta} m_{u d}^{1 / \delta}+\text { analytic } & T=T_{c} \\ c_{1} m_{u d} / a^{2}+\text { analytic } & T>T_{c}\end{cases}
$$

The ultraviolet singularity appears in perturbation theory at the one-quark-loop level. It is temperature independent. The infrared singularity occurs in the chirally broken phase $T<T_{c}$ [ [7]. It is seen in chiral perturbation theory at the one-pion-loop level. A square root is the thermal analog of a chiral $\log$. At $T_{c}$ we have the expected critical behavior.

The ultraviolet singularity in this quantity poses a problem for the continuum limit. Since the condensates for all flavors have this singularity, taking an the appropriate linear combination of 


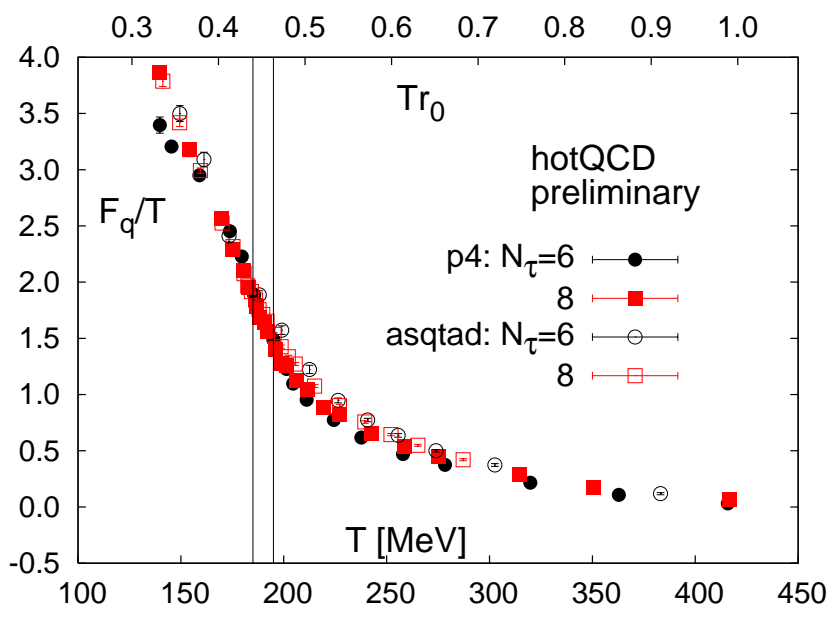

Figure 5: Renormalized screening free energy of a static quark (from the renormalized Polyakov loop) vs. temperature in $\mathrm{MeV}$ units (bottom scale) and $r_{0}$ units (top scale) for $N_{\tau}=6$ and 8 from a HotQCD study comparing p 4 fat 3 and asqtad staggered fermion formulations [5, 30]. Measurements are taken along a line of constant physics with $m_{u d}=0.1 m_{s}$.

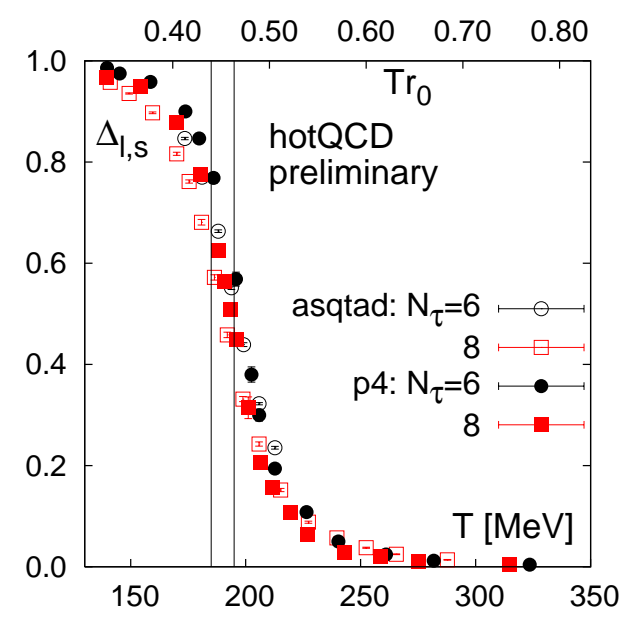

Figure 6: Chiral condensate difference ratio $v s$. temperature in $\mathrm{MeV}$ units (bottom scale) and $r_{0}$ units (top scale) for $N_{\tau}=6$ and 8 from a HotQCD study comparing p4fat 3 and asqtad staggered fermion formulations [5, 30]. Measurements are taken along a line of constant physics with $m_{u d}=0.1 m_{s}$.

light and strange quark condensates removes it, and dividing by the zero-temperature value removes the multiplicative renormalization factor [32]:

$$
D_{\ell, s}(T)=\left.\langle\bar{\psi} \psi\rangle\right|_{\ell}-\left.\frac{m_{\ell}}{m_{s}}\langle\psi \psi\rangle\right|_{s} \quad \Delta_{\ell, s}(T)=D_{\ell, s}(T) / D_{\ell, s}(T=0)
$$

The difference ratio $\Delta_{\ell, s}$ is shown in Fig. 6. We see a dramatic drop in this quantity over approximately the same temperature range over which we saw a rapid rise in Fig. $甘$. 


\subsubsection{Chiral susceptibility}

The chiral susceptibility measures fluctuations in the chiral condensate. Loosely speaking it is

$$
\chi=\partial\langle\bar{\psi} \psi\rangle(a, m, T) / \partial m
$$

where it is customary to distinguish the connected and disconnected contributions in terms of the Dirac matrix $M$ :

$$
\begin{aligned}
\chi_{\text {disc }} & =\frac{T}{V}\left[\left\langle\left(\operatorname{Tr} M^{-1}\right)^{2}\right\rangle-\left\langle\operatorname{Tr} M^{-1}\right\rangle^{2}\right] \\
\chi_{\text {conn }} & =-\frac{T}{V}\left\langle\operatorname{Tr} M^{-2}\right\rangle .
\end{aligned}
$$

Tradition holds that a peak in $\chi_{\text {disc }}$ marks the crossover, but see the discussion of possible distortions below.

Figure 7 shows recent results for the disconnected susceptibility. The peak occurs in roughly the same temperature range as the dramatic changes seen in the previous observables in Figs. 4 and 6. The peak height increases as expected as the light quark mass is decreased.

The first exploratory $N_{\tau}=8$ results for the domain wall action [4] are shown in Fig. 8. Because domain wall calculations are vastly more expensive, the domain wall effort is certainly not as advanced as other efforts. The $L_{s}=96$ study was undertaken to assure a small residual quark mass over the range of couplings shown.

The derivative in Eq (3.11) generates the correlator of the relevant chiral condensates, integrated over the space-time volume:

$$
\begin{aligned}
\chi & =C(p=0, T)=\int d^{4} x C(x, T) \\
C(x, T) & =\langle\bar{\psi} \psi(x) \bar{\psi} \psi(0)\rangle
\end{aligned}
$$

To be more precise, the derivative in the definition of the susceptibility (3.11) can involve any of the flavor condensates and any of the quark masses. For the light quark condensates, it is useful to distinguish the isosinglet and isotriplet chiral condensates, according to the isospin content of the operators in the correlator. These quantities are linear combinations of the disconnected and connected susceptibilities, namely, $\chi_{\text {sing }}=\chi_{\text {disc }}+2 \chi_{\text {conn }}$ and $\chi_{\text {trip }}=2 \chi_{\text {conn }}$.

The ultraviolet and infrared singularities of the isosinglet chiral susceptibility can be easily inferred from Eq (3.9) [7]:

$$
\chi_{\text {sing }} \sim \begin{cases}c_{1} / a^{2}+c_{1 / 2}(a, T) /(2 \sqrt{m})+\text { analytic } & T<T_{c} \\ c_{1} / a^{2}+c_{\delta} m^{1 / \delta-1}+\text { analytic } & T=T_{c} \\ c_{1} / a^{2}+\text { analytic } & T>T_{c}\end{cases}
$$

We see that it also suffers from an ultraviolet divergence, which makes it increasingly noisy as the lattice spacing is decreased. The infrared singularity at zero quark mass in the chirally broken phase $\left(T<T_{c}\right)$ arises from the vanishing of the pion mass in that limit. For this reason at small quark mass we should expect not only a peak at the transition point, but we should expect the susceptibility to grow at lower temperatures as well [7]. 

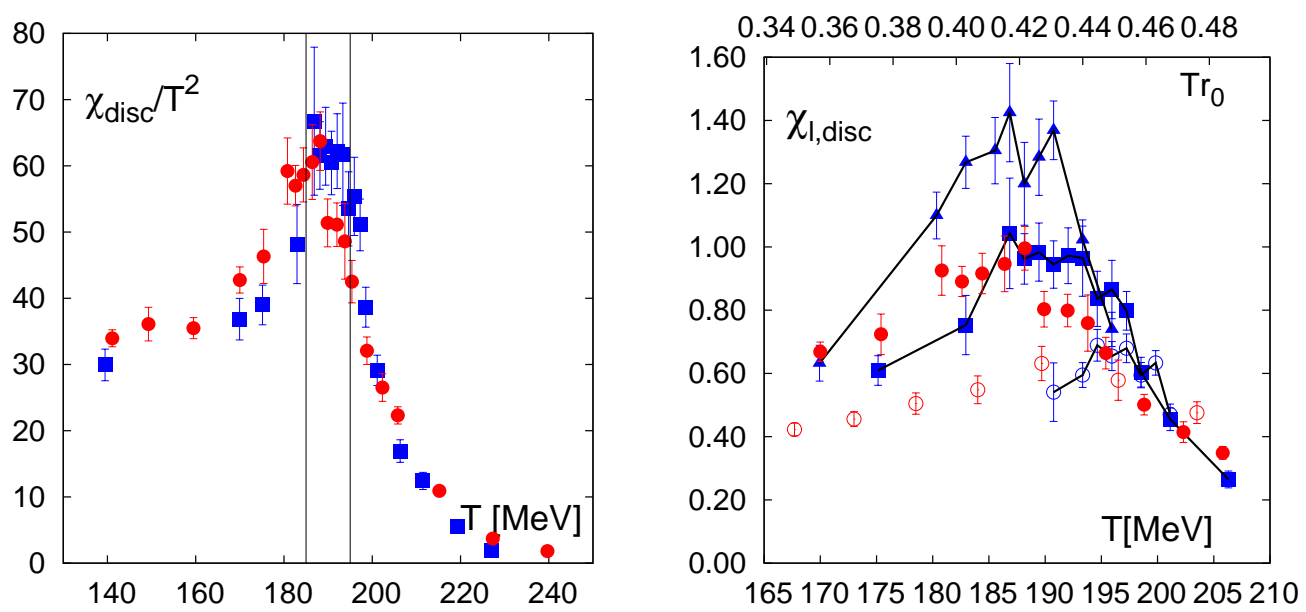

Figure 7: Left panel: Disconnected light quark susceptibility vs. temperature in MeV units (bottom scale). Right panel: closeup of the peak region. Lines merely connect the points. Red symbols, asqtad fermions. Blue symbols, p4fat3. Filled squares and circles are along a line of constant physics with $m_{u d}=0.1 m_{s}$. Open circles, with $m_{u d}=0.2 m_{s}$, filled triangles with $m_{u d}=0.05 m_{s}$. All results are HotQCD preliminary [5, 6, 30].

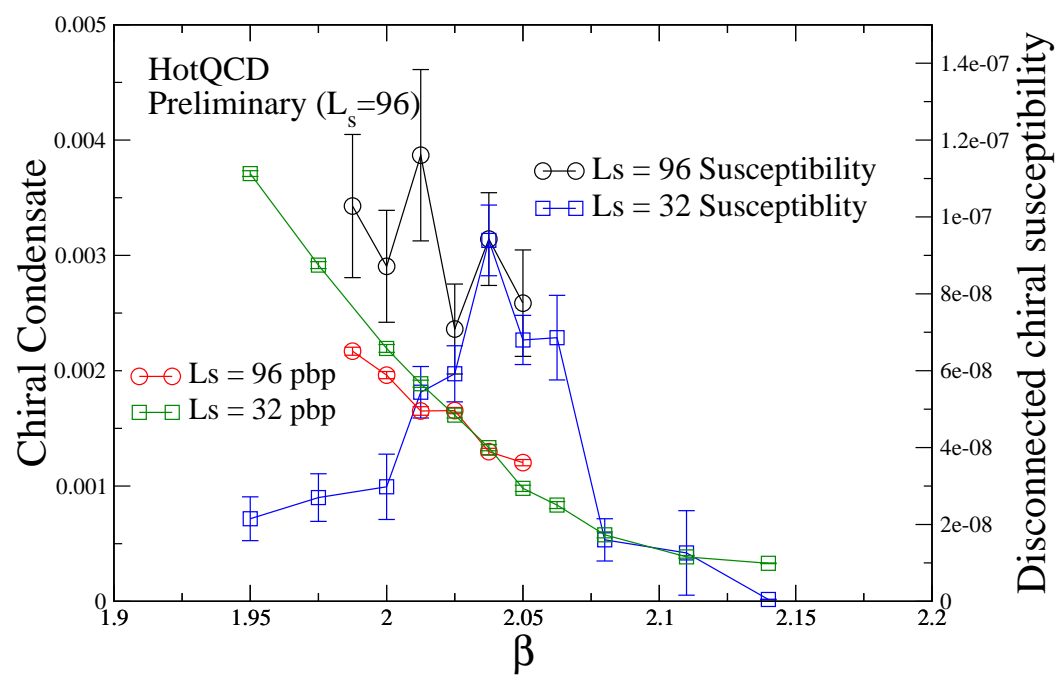

Figure 8: Chiral condensate (left scale) and disconnected chiral susceptibility (right scale) vs. the gauge coupling parameter $\beta$. Results are from a HotQCD study of $2+1$ flavor domain wall thermodynamics at $N_{\tau}=8$ for two choices of $L_{s}$ [ [†. Measurements are taken with light and strange quark masses fixed in lattice units $\left(a m_{u d}=0.003\right.$ and $\left.a m_{s}=0.37\right)$. 


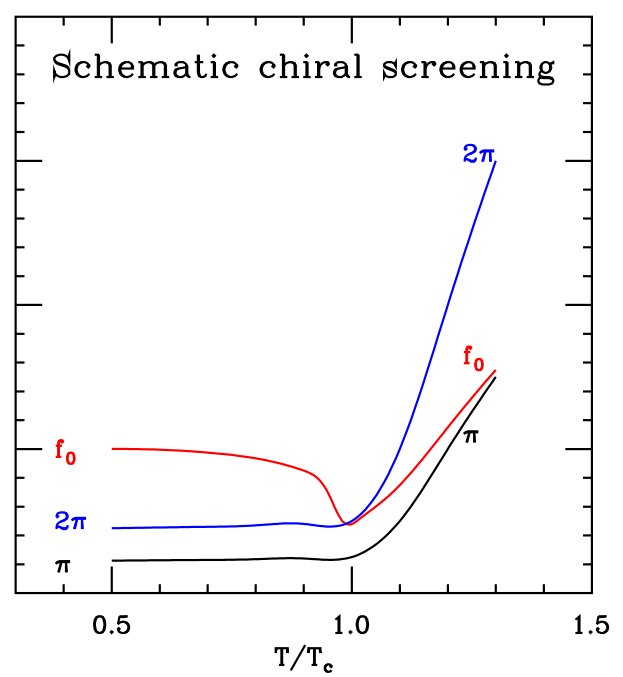

Figure 9: Sketch of the expected behavior of the light screening spectrum for the $\pi, f_{0}$ and $2 \pi$ threshold $v s$. temperature in units of the crossover temperature $T_{c}$.

To remove the ultraviolet singularity the Budapest/Wuppertal group subtracts the zero temperature value and multiplies by the square of the quark mass to cancel multiplicative renormalization factors:

$$
m_{q}^{2}\left[\chi\left(m_{q}, T\right)-\chi\left(m_{q}, 0\right)\right] / T^{4}
$$

Compared with the uncorrected susceptibility, along a line of constant physics away from the chiral limit, this definition tends to shift a peak to lower $T$ because $m_{q}^{2} / T^{2}$ decreases with increasing $T$. If instead of a peak, there is a shoulder, it might induce a peak.

\subsubsection{Screening masses as indicators of the transition}

From Eq (3.13) we see that a spectral component of mass $M(T)$ and weight $\rho(M, T)$ in the correlator contributes $\rho(M, T) / M(T)^{2}$, which is singular when $M(T)$ vanishes. Since the correlator is integrated over imaginary time, we can analyze the spatial dependence of the zero-Matsubarafrequency correlator to determine its spectrum. The masses in that case are "screening masses" [36]. Figure 9 sketches a possible scenario for the temperature dependence of the low spectral components of the isosinglet chiral condensate. In the chiral limit the $f_{0}$ must be degenerate with the pion for $T>T_{c}$, so for a continuous transition, it must drop to zero there. As the light quark mass is decreased, the two-pion threshold also vanishes, leading to an infinite chiral susceptibility for $T<T_{c}$ as well, as reflected in Eq (3.14). Close to the chiral limit, instead of a peak marking the transition, one might expect a cliff.

At this conference on behalf of the RBC/Bielefeld collaboration Laermann reported new measurements of screening masses in the scalar and vector isotriplet channels [37]. Some of their results are shown in Fig. 10. We see that the $\pi$ shows the behavior sketched in Fig. 9. Results for the $f_{0}$ are not available, but the $a_{0}$ shows a steep dip at the crossover.

As we see, screening masses may prove to be a useful indicator of the chiral transition, since they do not suffer from ultraviolet or chiral divergences, and they require no renormalization. 

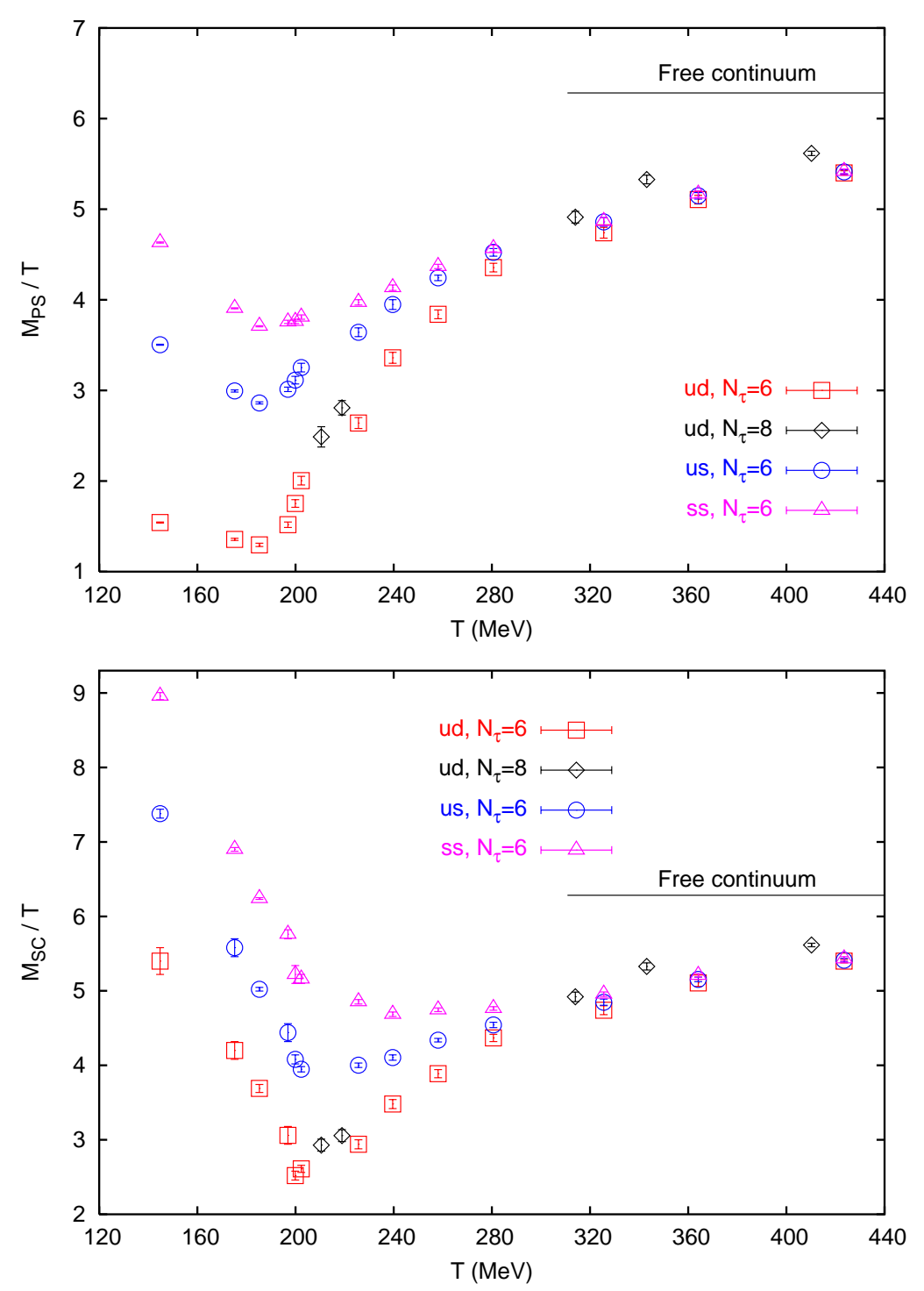

Figure 10: Screening masses for the pseudoscalar channel (upper panel) and scalar channel (lower panel) vs. temperature in a dynamical $2+1$ flavor simulation with p4fat 3 staggered fermions [38]. Measurements were taken along lines of constant physics with $m_{\pi} \sim 220 \mathrm{MeV}, m_{K}=500 \mathrm{MeV}$ and $N_{\tau}=6$ and 8 [37].

\subsection{Scale determination}

To quote the transition temperature $T_{c}$ in physical units requires a scale determination. The $\mathrm{Bu}-$ dapest/Wuppertal group favors setting the lattice scale with $f_{K}$, whereas MILC and RBC/Bielefeld use the Sommer parameter $r_{0}$ or the related $r_{1}$. At current typical lattice spacings and quark masses in staggered fermion simulations the $f_{K}$ scale results in a 10 to $20 \%$ lower temperature in $\mathrm{MeV}$ than the $r_{0}$ scale. This discrepancy vanishes at the physical quark mass and in the continuum. We should choose the scale so that the crossover temperature scales well. Of course, given the ambiguities in determining the crossover temperature, even in lattice units, that is an imprecise condition. The 
deconfinement-type variables are more useful to phenomenology. For them the $r_{0}$ scale seems to give reasonably consistent results as the lattice spacing is decreased, as we can see from Figs. 4 and 6 and Aoki et al. [29] (Fig. 4). Thus there appears to be no reason to abandon the $r_{0}$ scale for now.

In the past two years there have been some seemingly contradictory estimates of the transition temperature. Aoki et al. reported that at physical quark masses in the continuum, $T_{c}=151$ (3)(3) $\mathrm{MeV}$ from a peak in the chiral susceptibility and $T_{c}=175(2)(4)$ from the inflection point in the quark number susceptibility and the Polyakov loop variable. These are to be compared with an older result from the MILC collaboration 169(12)(4) MeV based on the chiral susceptibility [39] and a more recent determination of 192(7)(4) MeV by the Bielefeld/RBC group based on a combination of chiral and deconfining observables [40]. The last two groups used the $r_{0}$ (or $r_{1}$ ) scale. The Budapest/Wuppertal group has carefully listed sources of the discrepancy, which include ambiguities in locating the crossover, their preferred renormalization of the chiral susceptibility, and their preference for the $f_{K}$ scale. This year we can add to the list the possibility that the chiral susceptibility develops an asymmetric peak or shoulder, which would be even more sensitive to the renormalization procedure and should not be modeled by a parabola. A combination of these effects could certainly account for the discrepancy.

\section{Equation of state}

The equation of state is fundamental to hydrodynamic calculations of the expansion of hot hadronic matter.

\subsection{Standard integral method}

The currently popular method for calculating the equation of state begins with the latticethermodynamic identity at fixed $N_{\tau}$, which expresses the trace of the energy momentum tensor or "interaction measure" $I$ in terms of the derivative of the $\log$ of the partition function $Z$ :

$$
I=\varepsilon-3 p=-\frac{T}{V} \frac{d \ln Z}{d \ln a} .
$$

The derivative with respect to lattice spacing is taken with fixed output hadron masses. Thus it involves the derivative of the bare lattice parameters, i.e., the gauge coupling $g$, quark masses, and for some actions the tadpole coefficient $u_{0}$, with respect to the cutoff scale and the expectation values of the action operators. All of these nonperturbative quantities are readily calculated in lattice simulations.

We normalize the energy and pressure to zero at zero temperature. This eliminates an ultraviolet divergence of order $1 / a^{4}$. This is done by subtracting the zero temperature quantity, calculated with the same bare parameters. Because we are subtracting two ultraviolet singular quantities, we must increase the simulation sample size dramatically as the lattice spacing is decreased. The calculational cost thus grows steeply as we approach the continuum limit. In what follows we will assume this subtraction has been done for all thermodynamic quantities.

A second identity becomes

$$
\frac{p}{T}=\left.\frac{\partial \ln Z}{\partial V}\right|_{T} \rightarrow(\ln Z) / V
$$



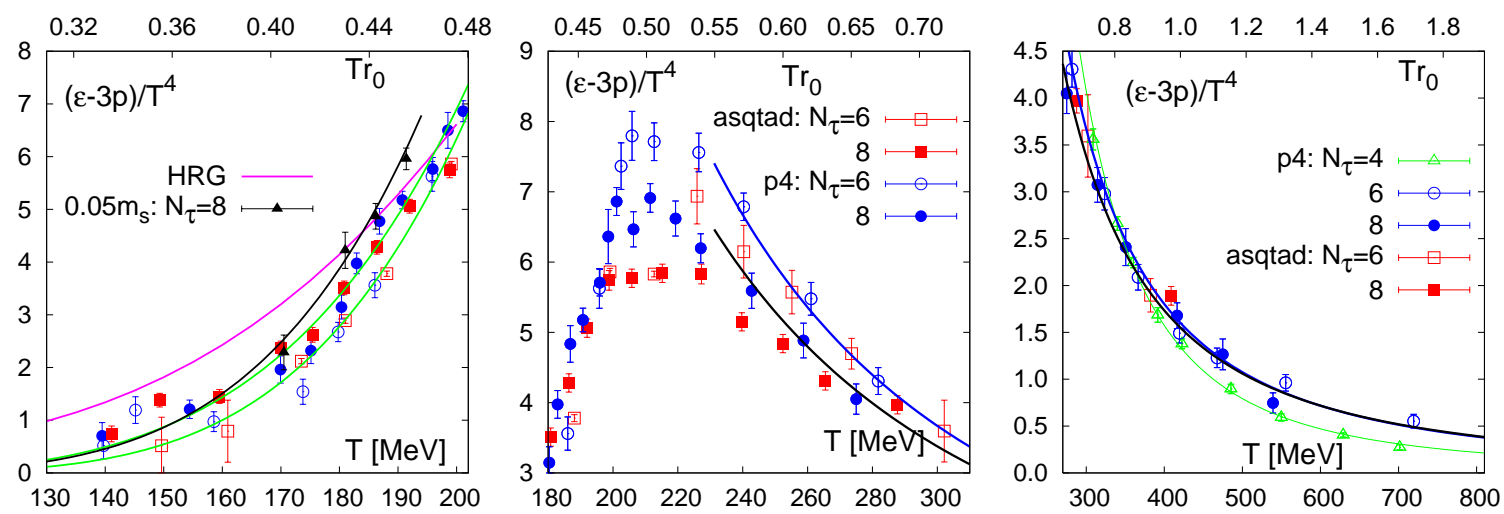

Figure 11: Details of the dependence of the interaction measure on temperature in $\mathrm{MeV}$ units (bottom scale) and $r_{0}$ units (top scale) for three temperature ranges left to right: low, mid, and high, for $N_{\tau}=6$ and 8 from a HotQCD study comparing p4fat3 and asqtad staggered fermion formulations [5, 6, 30]. Measurements in most cases are taken along a line of constant physics with $m_{u d}=0.1 m_{s}$. Results in the high temperature range at $N_{\tau}=4$ are from [38]. In the low temperature range the magenta curve is the prediction of a hadron resonance gas model. The other curves in that range are spline fits to the data. The curves in the high temperature range are fits to a quadratic in $1 / T^{2}$.

in the thermodynamic limit for which $\ln Z \propto V$. Finite-size deviations from this limit could produce deviations from the Stefan-Boltzmann law [41, 42]. Such finite size effects may be important at ultrahigh $T$, where we would like to compare with perturbation theory [43].

Putting Eqs (4.1) and (4.2) together gives the the pressure as the integral of the interaction measure from coarse to fine lattice spacing, i.e., low to high temperature:

$$
\left.\frac{V p}{T}\right|_{a}-\left.\frac{V p}{T}\right|_{a_{0}}=-\int_{\ln a_{0}}^{\ln a} \frac{V^{\prime}}{T^{\prime}}\left(\varepsilon^{\prime}-3 p^{\prime}\right) d \ln a^{\prime} .
$$

If the lower limit is sufficiently low in temperature, the pressure is zero. With pressure and interaction measure in hand we immediately get the energy density and entropy.

Recent results for the interaction measure for $N_{\tau}=6$ and 8 are shown in Fig. 11. The resulting equation of state and pressure were shown in Fig. 7 and the entropy density is shown in Fig. 12. Whether there is a statistically significant disagreement between the asqtad and p4fat 3 results in the central region remains to be determined after further data are accumulated. In the high temperature range $T \in[250,700] \mathrm{MeV}$, the results can be fit to

$$
(\varepsilon-3 p) / T^{4}=b / T^{2}+c / T^{4}
$$

There seems to be no need yet to include perturbative $1 / \log T$ terms in the fit coefficients $b$ and $c$. The plot in the low temperature range compares the lattice result with predictions of a hadron resonance gas model. Since the lattice calculations include cutoff effects, it would be premature to draw conclusions based on a disagreement at this level.

\subsection{New $T$ integral method}

In the past year the WHOT collaboration introduced a new method in which the integral over 


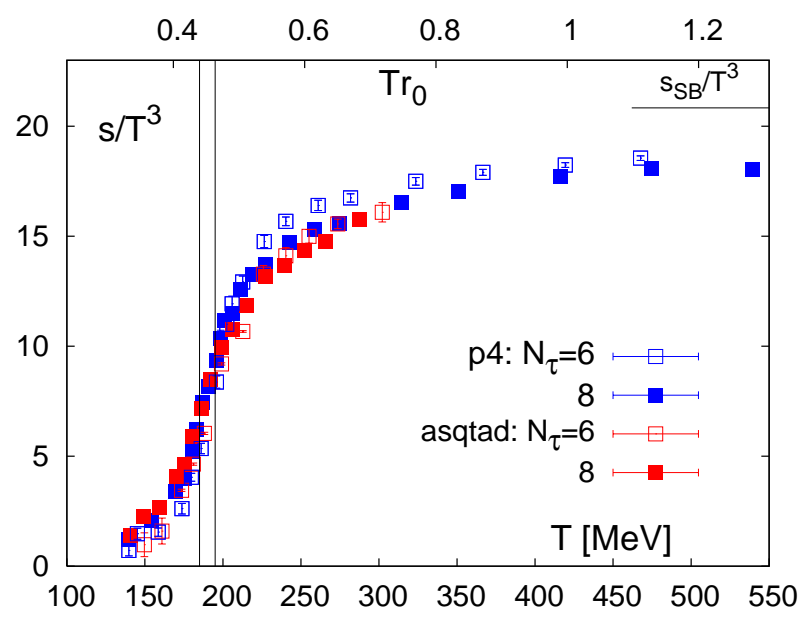

Figure 12: Entropy density divided by the third power of the temperature $v s$. temperature in $\mathrm{MeV}$ units (bottom scale) and $r_{0}$ units (top scale). for $N_{\tau}=6$ and 8 from a HotQCD study comparing p4fat 3 and asqtad staggered fermion formulations 汭, 30. Measurements in most cases are taken along a line of constant physics with $m_{u d}=0.1 m_{s}$.

lattice spacing at fixed $N_{\tau}$ is replaced by an approximate integral over $N_{\tau}$ at fixed lattice spacing (i.e., fixed bare lattice parameters) [8, 44].

The method starts from an alternative form of Eq (4.1) with Eq (4.2):

$$
I / T^{4}=\frac{d\left(p / T^{4}\right)}{d \ln T} .
$$

The pressure is then computed by integrating the interaction measure with respect to $\ln T$ or equivalently $\ln \left(N_{\tau}\right)$. Since $N_{\tau}$ is an integer, to reduce discretization errors in the sampling of the integrand, one must reduce the temporal lattice spacing $a_{t}$. An anisotropic lattice helps.

With this potentially computationally cheaper method the zero temperature subtraction is common to all $N_{\tau}$, and with bare lattice parameters fixed, one necessarily works along lines of constant physics. Figure 13 shows the result of a test calculation done for pure SU(3) Yang-Mills theory.

\section{Plasma Structure}

In addition to the phase diagram and equation of state, lattice simulations provide information about the structure of hot hadronic matter. I mention two recent developments: a new effort to determine the shear and bulk viscosity over a range of temperatures and a new study of the spatial string tension.

\subsection{Transport Coefficients}

Analysis of RHIC heavy ion collisions suggests that high temperature hadronic matter is an exceptionally good fluid. To confirm this hydrodynamical characterization requires computing the transport coefficients, namely the shear $(\eta)$ and bulk $(\zeta)$ viscosities. They are obtained from 


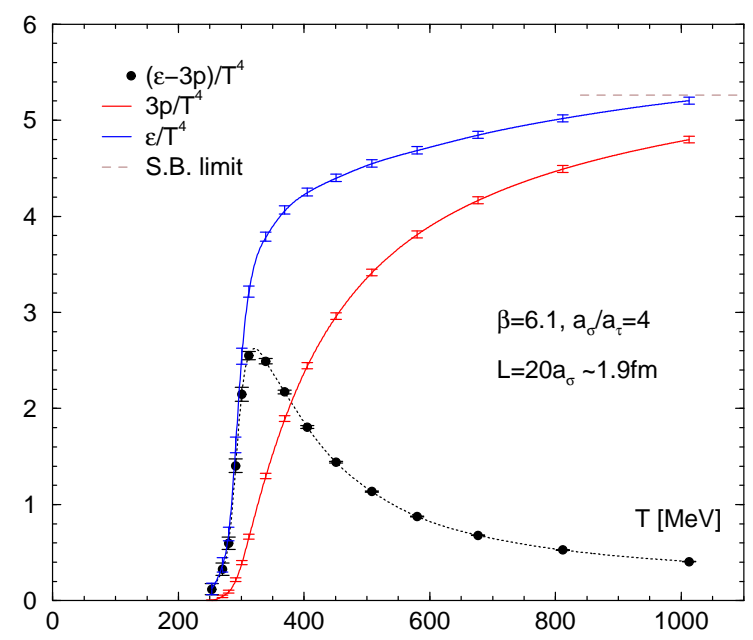

Figure 13: Equation of state (interaction measure, energy density and pressure) for pure Yang-Mills theory, obtained using the $T$ integral method at fixed lattice spacing $a_{\sigma}=0.097 \mathrm{fm}$ and aspect ratio $a_{\tau} / a_{\sigma}=4$ [8, 44].

correlators of the energy-momentum tensor at temperature $T$

$$
C\left(x_{0}, \mathbf{x}, T\right)=\left\langle T_{\mu \nu}\left(x_{0}, \mathbf{x}\right) T_{\rho \sigma}(0) .\right\rangle
$$

We need its spectral function $\rho$, which we obtain from the Kubo formula

$$
C\left(x_{0}, \mathbf{q}, T\right)=\int_{0}^{\infty} d \omega \rho(\omega, \mathbf{q}, T) \frac{\cosh \omega\left(x_{0}-1 / 2 T\right)}{\sinh (\omega / 2 T)} .
$$

The transport coefficients are obtained from the low-frequency behavior of the spectral function

$$
\eta(T)=\pi \lim _{\omega \rightarrow 0} \frac{\rho_{12,12}(\omega, 0, T)}{\omega} \quad \zeta(T)=\frac{\pi}{9} \lim _{\omega \rightarrow 0} \frac{\rho_{i i, j j}(\omega, 0, T)}{\omega}
$$

This has been a well known challenging problem since it was first attempted by Karsch and Wyld [45]. The correlator is noisy, requiring high statistics. Going from a Euclidean correlator $C\left(x_{0}\right)$ to $\rho(\omega)$ is a very difficult inverse problem. Because of time-reflection symmetry, a simulation at $N_{\tau}=8$ has only five, typically noisy, independent values.

Possible remedies include (1) assuming a functional form for $\rho$ and fitting its parameters, (2) decreasing the time interval $a_{t}$, allowing a larger $N_{\tau}$, and (3) adding further constraints on $\rho$, such as maximum entropy.

Meyer [9, 46, 47] has done a new high statistics calculation in pure Yang-Mills theory and uses a paramerization of the spectral function in terms of an optimized basis set that folds in appropriate perturbative behavior. For the ratio of shear viscosity to entropy density, he finds $\eta / s=0.134$ (33) at $1.65 T_{c}$ where perturbation theory gives 0.8 , and for the ratio of bulk viscosity to entropy density, $\zeta / s<0.15$ at $1.65 T_{c}$ and $\zeta / s<0.015$ at $3.2 T_{c}$.

\subsection{Spatial string tension}

Despite its popular characterization as deconfined, high temperature hadronic matter retains vestiges of confinement. Space-like Wilson loops still exhibit the area-law behavior associated 


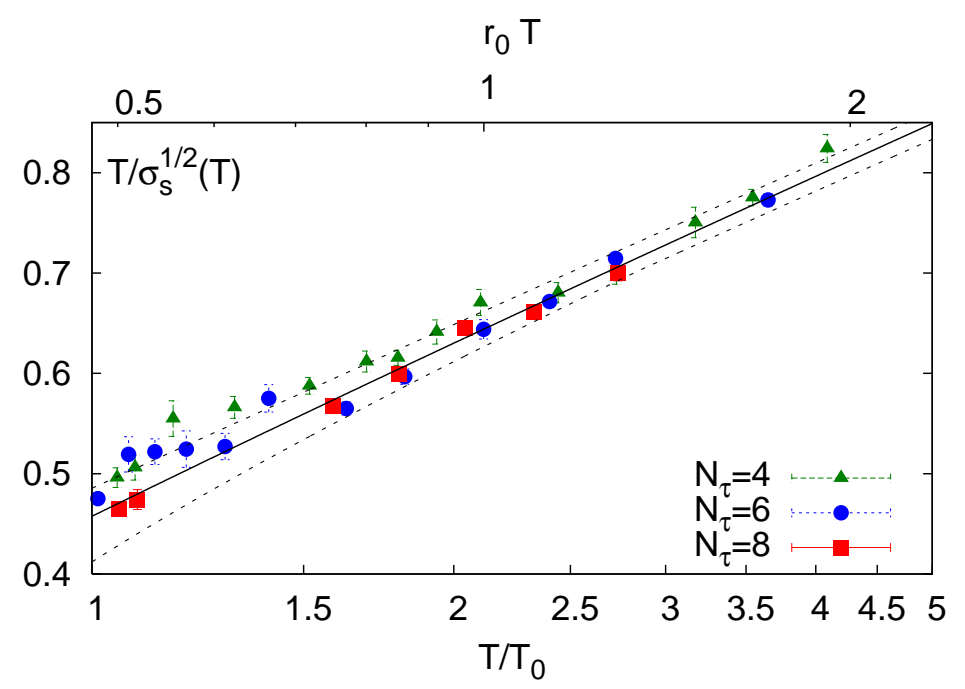

Figure 14: Temperature divided by the square root of the spatial string tension $\sigma_{s} v s$. temperature in units of the crossover temperature $T_{0}$ (lower scale) and in $r_{0}$ units (upper scale) for $2+1$ flavors of p4fat 3 quarks on lattices with $N_{\tau}=4,6$ and 8 . The solid curve (with uncertainties indicated by the dashed lines) is the prediction of the dimensionally reduced theory [10]

with confinement. This is readily seen by considering dimensional reduction, in which for $T \gg T_{c}$ the short Euclidean time dimension is collapsed, leaving three spatial dimensions, one of which is reinterpreted as the Euclidean time coordinate of a $2+1$ dimensional field theory.

The reduction of QCD has these characteristics:

- Quarks acquire a large 3D mass $\sqrt{(\pi T)^{2}+m_{q}^{2}}$

- The fourth component of the color vector potential $A_{0}$ becomes a scalar field, and we get a confining gauge-Higgs theory.

- The 3D and 4D gauge couplings are related through $g_{3}=g_{4} \sqrt{T}$.

- The spatial Wilson loop gives the 3D potential and 3D string tension.

In a recent calculation Cheng et al compared the spatial string tension of the full 4D theory with its predicted behavior in 3D perturbation theory [10]. The comparison is shown in Fig. 14. The good agreement with perturbation theory at temperatures as low as $1.5 T_{c}$ is unexpected.

\section{Conclusions}

In a reasonably well-matched calculation, new high statistics results from HotQCD show good agreement between two different staggered fermion formulations, i.e., p4fat 3 and asqtad. Not surprisingly, simulations with these inexpensive algorithms are more advanced than those with other fermion actions, as we have seen from the first exploratory domain-wall-fermion simulations at $N_{\tau}=8$ with a quite small residual quark mass. To make progress we need to understand the 
importance of cutoff effects and to come closer to the physical point. Calculations with other fermion actions can provide an important check, but those actions must be improved at least to the same level as the staggered fermion actions before they can play this role effectively.

We are learning more about the phase structure of zero-baryon-density QCD as a function of the light quark masses, but these results are especially sensitive to cutoff effects. More work is still needed.

There has been recent progress in methodology. The WHOT collaboration has developed a new method for determining the equation of state, and Meyer has proposed new methods for determining transport coefficients.

Finally, in measurements of the spatial string tension, we have seen interesting agreement with predictions of dimensional reduction.

\section{Acknowledgments}

I am grateful to my many colleagues for providing figures and assisting in identifying novel work. I thank Rajan Gupta, Urs Heller, and Ludmila Levkova for helpful comments about the manuscript. This review is supported by grants from the US Department of Energy and US National Science Foundation.

\section{References}

[1] R. C. Hwa and X. N. Wang, eds. Quark-gluon plasma. Vol. 3, River Edge, USA: World Scientific (2004) $777 \mathrm{p}$.

[2] M. G. Alford, K. Rajagopal, and F. Wilczek, Color-flavor locking and chiral symmetry breaking in high density QCD, Nucl. Phys. B537 (1999) 443-458, [hep-ph/9804403].

[3] S. Ejiri, Recent progress in lattice QCD at finite density, BOS (LAT2008) 002.

[4] M. Cheng et al., QCD Thermodynamics from domain wall fermions, POS (LAT2008) 180.

[5] R. Gupta et al., The EOS from simulations on BlueGene L supercomputer at LLNL and NYBlue, POS (LAT2008) 170.

[6] W. Soeldner, Quark mass dependence of the QCD equation of state on Nt=8 lattices, POS (LAT2008) 173.

[7] F. Karsch, Fluctuation of Goldstone modes and the chiral transition in QCD in Proceedings of the Conference on Strong and Electroweak Matter (SEWM), Amsterdam, 26-29 August 2008, Nucl. Phys. $A$ (to be published, 2008).

[8] T. Umeda et al., Thermodynamics of SU(3) gauge theory at fixed lattice spacing, POS (LAT2008) 174.

[9] H. Meyer, Energy-momentum tensor correlators and viscosity, BOS (LAT2008) 017

[10] M. Cheng et al., The spatial string tension and dimensional reduction in QCD, Phys. Rev. D78 (2008) 034506, [0806.3264].

[11] A. Deuzeman, The physics of eight flavours, BOS (LAT2008) 060.

[12] K. Szabó, The QCD transition with 2+1 dynamical flavors, PoS (LAT2008) 207. 
[13] C. W. Bernard et al., The QCD spectrum with three quark flavors, Phys. Rev. D64 (2001) 054506, [hep-lat/0104002].

[14] U. M. Heller, F. Karsch, and B. Sturm, Improved staggered fermion actions for QCD thermodynamics, Phys. Rev. D60 (1999) 114502, [hep-lat/9901010].

[15] Y. Aoki, Z. Fodor, S. D. Katz, and K. K. Szabó, The equation of state in lattice QCD: With physical quark masses towards the continuum limit, JHEP 01 (2006) 089, [hep-lat/ 0510084 ].

[16] P. Hegde, F. Karsch, E. Laermann, and S. Shcheredin, Lattice cut-off effects and their reduction in studies of QCD thermodynamics at non-zero temperature and chemical potential, Eur. Phys. J. $\mathbf{C 5 5}$ (2008) 423-437, [0801.4883].

[17] W. Bietenholz, R. Brower, S. Chandrasekharan, and U. J. Wiese, Progress on perfect lattice actions for QCD, Nucl. Phys. Proc. Suppl. 53 (1997) 921-934, hep-lat/9608068.

[18] R. V. Gavai and S. Sharma, Towards QCD thermodynamics using exact chiral symmetry on the lattice, 0805.2866 .

[19] N. Ishizuka, M. Fukugita, H. Mino, M. Okawa, and A. Ukawa, Operator dependence of hadron masses for Kogut-Susskind quarks on the lattice, Nucl. Phys. B411 (1994) 875-902.

[20] E. Laermann and O. Philipsen, Status of lattice QCD at finite temperature, Ann. Rev. Nucl. Part. Sci. 53 (2003) 163-198, hep-ph/0303042.

[21] R. D. Pisarski and F. Wilczek, Remarks on the chiral phase transition in chromodynamics, Phys. Rev. D29 (1984) 338-341.

[22] M. D’Elia, A. Di Giacomo, and C. Pica, Two flavor QCD and confinement, Phys. Rev. D72 (2005) 114510, [hep-lat/0503030].

[23] J. B. Kogut and D. K. Sinclair, Evidence for $O(2)$ universality at the finite temperature transition for lattice QCD with 2 flavours of massless staggered quarks, Phys. Rev. D73 (2006) 074512, [hep-lat/0603021].

[24] Y. Aoki, G. Endrödi, Z. Fodor, S. D. Katz, and K. K. Szabó, The order of the quantum chromodynamics transition predicted by the standard model of particle physics, Nature 443 (2006) 675-678, [hep-lat/0611014.

[25] F. Karsch, E. Laermann, and C. Schmidt, The chiral critical point in 3-flavor QCD, Phys. Lett. B520 (2001) 41-49, [hep-lat/0107020].

[26] F. Karsch et al., Where is the chiral critical point in 3-flavor QCD?, Nucl. Phys. Proc. Suppl. 129 (2004) 614-616, [hep-lat/0309116].

[27] P. de Forcrand and O. Philipsen, The chiral critical line of $N(f)=2+1 Q C D$ at zero and non-zero baryon density, JHEP 01 (2007) 077, hep-lat/0607017].

[28] P. de Forcrand and O. Philpsen, The curvature of the critical surface $\left(m_{u d}, m_{s}\right)^{\text {crit }}(m u)$, on finer and bigger lattices, POS (LAT2008) 208.

[29] Y. Aoki, Z. Fodor, S. D. Katz, and K. K. Szabó, The QCD transition temperature: Results with physical masses in the continuum limit, Phys. Lett. B643 (2006) 46-54, [hep-lat/0609068].

[30] HotQCD Collaboration, C. DeTar and R. Gupta, Toward a precise determination of $T_{c}$ with $2+1$ flavors of quarks, POS (LAT2007) 179, 0710.1655.

[31] C. Aubin et al., Light hadrons with improved staggered quarks: Approaching the continuum limit, Phys. Rev. D70 (2004) 094505, [hep-lat/ 0402030 ]. 
[32] F. Karsch, Recent lattice results in finite temperature and density QCD, part II, PoS (LAT2007) 015, 0711.0661.

[33] Y. Hatta and T. Ikeda, Universality, the QCD critical / tricritical point and the quark number susceptibility, Phys. Rev. D67 (2003) 014028, hep-ph/0210284.

[34] C. W. Bernard et al., Two-flavor staggered fermion thermodynamics at $N(t)=12$, Phys. Rev. D54 (1996) 4585-4594, [hep-lat/9605028].

[35] O. Kaczmarek, F. Karsch, F. Zantow, and P. Petreczky, Static quark anti-quark free energy and the running coupling at finite temperature, Phys. Rev. D70 (2004) 074505, [hep-lat/0406036].

[36] C. E. DeTar and J. B. Kogut, The Hadronic spectrum of the quark plasma, Phys. Rev. Lett. 59 (1987) 399.

[37] E. Laermann et al., Recent results on screening masses, POS (LAT2008) 193.

[38] M. Cheng et al., The QCD Equation of state with almost physical quark masses, Phys. Rev. D77 (2008) 014511, [0710.0354].

[39] MILC Collaboration, C. Bernard et al., QCD thermodynamics with three flavors of improved staggered quarks, Phys. Rev. D71 (2005) 034504, [hep-lat/ 0405029 ].

[40] M. Cheng et al., The transition temperature in QCD, Phys. Rev. D74 (2006) 054507, [hep-lat/0608013].

[41] F. Gliozzi, The Stefan-Boltzmann law in a small box and the pressure deficit in hot SU(N) lattice gauge theory, J. Phys. A40 (2007) F375-4922, hep-lat/0701020.

[42] M. Panero, Geometric effects in lattice QCD thermodynamics, PoS (LAT2008) 175.

[43] G. Endrödi, Z. Fodor, S. D. Katz, and K. K. Szabó, The equation of state at high temperatures from lattice QCD, BOS (LAT2007) 228, [0710.4197].

[44] T. Umeda et al., Fixed scale approach to equation of state in lattice $Q C D, 0809.2842$.

[45] F. Karsch and H. W. Wyld, Thermal Green's functions and transport coefficients on the lattice, Phys. Rev. D35 (1987) 2518.

[46] H. B. Meyer, A calculation of the bulk viscosity in SU(3) gluodynamics, Phys. Rev. Lett. 100 (2008) 162001, [0710.3717].

[47] H. B. Meyer, A calculation of the shear viscosity in SU(3) gluodynamics, Phys. Rev. D76 (2007) 101701, [0704.1801]. 